\title{
Actividad Eléctrica Asociada al Huracán Otto (2016) en el Mar Caribe y en el Corredor Seco Centroamericano
}

\author{
Dayanna Arce-Fernández ${ }^{1}$ (D) , Jorge A. Amador ${ }^{1}$ \\ ${ }^{1}$ Universidad de Costa Rica, Escuela de Física, Centro de Investigaciones Geofisicas, San José, \\ Costa Rica.
}

Recibido em: 2 de Mayo de 2019 - Aceptado em: 16 de Septiembre de 2019

\begin{abstract}
Resumen
Otto (2016) es el primer huracán en la historia documentada de ciclones tropicales de haber alcanzado directamente Costa Rica. Este trabajo analiza las descargas eléctricas (DE) de Otto en noviembre 2016 en su paso desde el Caribe hasta el Pacífico, usando datos de la World Wide Lightning Location Network; lo cual constituye una investigación pionera en esta región de Centroamérica. Las DE asociadas a Otto capturan muy bien las fases de intensificación y degradación de este huracán, que como variable podría ser parametrizada en los modelos numéricos para mejorar el entendimiento y evolución de estos sistemas. Otto presentó tres picos máximos de descargas; uno en categoría 1 en la escala Saffir-Simpson, otro en categoría 2 y el último en la categoría $3(\mathrm{OH} 3)$, poco antes de su entrada a tierra entre Costa Rica y Nicaragua. La región de mayor cantidad de DE se desplazó del centro hacia las zonas más externas del huracán conforme éste se intensificó. En el caso de $\mathrm{OH} 3$, el máximo de DE se ubicó en las bandas externas de Otto (250-300 km). Durante su evolución, las de máximas DE observadas estaban relacionadas con temperaturas del tope de las nubes menores a los $-70{ }^{\circ} \mathrm{C}$.
\end{abstract}

Palabras clave: Otto 2016, ciclón tropical, actividad eléctrica, WWLLN, Corredor Seco Centroamericano, Centroamérica.

\section{Electrical Activity Associated with Hurricane Otto (2016) in the Caribbean Sea and in the Central American Dry Corridor}

\begin{abstract}
Otto (2016) is the first hurricane in the documented tropical cyclone history to have directly reached Costa Rica. This work analyses the electrical discharges (ED) associated with Otto in November 2016 during its trajectory from the Caribbean to the Pacific, using data from the World Wide Lightning Location Network; which constitutes a pioneering research in the Central America region. Otto ED captured very well Ottoös intensification and degradation phases, which as a variable could be parametrized in numerical models to improve the knowledge and evolution of these systems. Otto presented three ED peaks during each one of the categories it attained; the last one during hurricane category 3 (Saffir-Simpson scale) just prior to make landfall between Costa Rica and Nicaragua. The region with most ED moved from the near center of the system to its external region in pace with the hurricane intensification. When Otto reached category 3, the maxima of ED was observed in Otto's external bands (250-300 km). During Otto's evolution ED maximum were observed to be related to cloud top temperatures of less than $-70{ }^{\circ} \mathrm{C}$.
\end{abstract}

Keywords: Otto 2016, tropical cyclone, electrical activity, WWLLN, Central America Dry Corridor, Central America.

\section{Introducción}

Las regiones tropicales del globo son fuente de gran cantidad de sistemas meteorológicos caracterizados por el desarrollo de convección profunda e intensa actividad eléctrica (e.g., Ribeiro et al., 2014; Albretch et al., 2016). La Zona de Convergencia Intertropical (ZCIT, Collier y
Hughes, 2007; Vincent et al., 2011), las oscilaciones intraestacionales (Serra et al., 2014), las ondas atmosféricas (ondas en los estes; Burpee, 1972) y los ciclones tropicales (Kuo, 1965; Lyons y Keen 1994; Molinari et al., 1999; Corbosiero et al., 2003; Pan et al., 2010; Abarca et al., 2011) son algunos ejemplos de sistemas atmosféri-

Autor de correspondencia: Dayanna Arce-Fernández, dayanna.arcefernandez@ucr.ac.cr. 
cos que exhiben características como las descritas arriba. La configuración de las variables básicas (temperatura, presión, humedad, etc.) en estos sistemas, así como los fenómenos físicos que representan la covariabilidad de esas variables (por ejemplo, la precipitación, la nubosidad y las descargas eléctricas) tienen distribuciones espaciales y temporales muy particulares que a menudo originan impactos sociales en diferentes escalas de movimiento.

Los ciclones tropicales (CT) causan daños ambientales muy severos al provocar inundaciones, deslaves, fuertes vientos y marejadas, entre otras calamidades. Las condiciones anómalas asociadas a los CT no solo tienen un elevado costo social por el número de muertos, heridos y desplazados, sino que generan además daños económicos considerables en infraestructura, puentes, caminos y sistemas eléctricos (Amador, 2011). La frecuencia anual o estacional de los CT en las cuencas del Caribe (Amador et al., 2010) y en el Pacífico tropical del este (PTE) está relacionada a su vez con otros modos atmosféricos de más baja frecuencia que afectan la región, como El Niño Oscilación del Sur (ENOS; Mora y Amador, 1999: Amador et al., 2016a, b) o la corriente en chorro del Caribe (CLLJ, por sus siglas en inglés; Amador, 1998, 2008). Las interacciones no lineales entre estos sistemas restringen la predictibilidad a corto plazo de los CT y como consecuencia la toma oportuna de decisiones de carácter socioeconómico. El estudio del origen, la estructura y la evolución de estos sistemas es entonces fundamental para minimizar algunos de los impactos que generan.

En las últimas décadas, el desarrollo científico y tecnológico ha hecho posible conocer por medio de observaciones directas (por ejemplo, aviones con instrumentación especial a bordo) e indirectas (teledetección), algunos de los atributos y la estructura de los sistemas tropicales. Diferentes métodos y tipos de datos han sido utilizados para el estudio de las características y evolución de estos sistemas meteorológicos, entre ellos, el uso de datos de reanálisis (Bengtsson et al., 2004; Murakami, 2014), modelos numéricos (Anthes, 1972; Kurihara and Tuley, 1974; Halperin et al., 2013), datos de observaciones in situ (Aberson et al., 2006; Elsberry and Harr, 2008) e información satelital (Mueller et al., 2006; Olander and Velden, 2007).

En el caso de los CT, se han realizado también estudios con las técnicas anteriores y en algunos casos con datos de descargas eléctricas (DE) para determinar la distribución de esa actividad en el ojo y en las bandas internas y externas de estos sistemas (Molinari et al., 1994, 1999; Corbosiero and Molinari, 2003; Solorzano et al., 2008; Abarca et al., 2011). Las plataformas de observación que proveen datos sobre la distribución de $\mathrm{DE}$, no solo han servido para el estudio de los CT y su evolución, sino que han sido también utilizados a nivel global para estudiar la actividad de rayos asociada a otros sistemas meteorológicos que generan impactos sociales y ambien- tales relevantes. Por ejemplo, las DE son la causa de las aproximadamente 2400 muertes y 240000 lesiones que ocurren globalmente (Raga et al., 2014). De acuerdo a Jensenius (2017), durante el periodo 2006-2016 un total de 352 muertes fueron atribuidas a descargas eléctricas únicamente en los Estados Unidos de América, de ellas buena parte sucedieron durante actividades de pesca, paseos en barco y actividades de campamento. En México, la distribución espacial de muertes está relacionada con la exposición a tormentas eléctricas, la agricultura tradicional y bajos niveles de educación (Raga et al., 2014). Así mismo, la caída de rayos puede ser el inicio de incendios forestales (Latham y Williams, 2001) y se ha logrado mostrar que influyen en los niveles de contaminación atmosférica (Kochtubajda et al., 2002). En Centroamérica (CA) solo en el 2018 fueron contabilizadas 28 muertes y 27 heridos por rayos, casi todos ellos se dieron mientras las personas estaban en campo abierto (Amador et al., 2019).

La teledetección ha sido utilizada en la región de interés de CA con diferentes propósitos, entre ellos, el estudio de los efectos geofísicos del terremoto de Limón, Costa Rica en 1991 (Amador et al., 1994), la caracterización de algunos manglares en la región (Lizano et al., 2001) y la distribución para un corto periodo de las DE en el huracán Otto 2016 (Amador et al., 2017). Fuera de esta zona, esta técnica se utilizó además para determinar la relación entre las DE y la erupción volcánica del complejo Cordón Caulle en Sur América en 2011 (Nicora et al., 2013). Estos dos últimos trabajos usaron datos de la red global World Wide Lightning Location Network (WWLLN, por sus siglas en inglés). En un estudio sobre los CT usando información de la WWLLN se asocia la distribución de DE a la intensidad o cambios en la intensidad de los huracanes y la distribución de convección (Molinari et al., 1994; Abarca et al., 2011). De este último trabajo se desprenden dos importantes conclusiones, la primera es que la densidad de rayos es un parámetro potencial para distinguir CT que pueden o no intensificarse y la segunda se refiere a que los datos de la WWLLN capturan muy bien la convección a pesar de la poca eficiencia de esta red. Las descargas eléctricas han mostrado ser una especie de proxi para detectar la convección en ciclones tropicales (Abarca et al., 2010), en especial en aquellos $\mathrm{CT}$ que tiene una rápida intensificación como es el caso de Otto que mostró nubosidad de gran desarrollo vertical del tipo Cumulonimbo entre las 0600 y 0900 UTC del día 24 de noviembre de 2016. El estudio del comportamiento de las DE en un CT es entonces importante no solo porque puede facilitar la comprensión del fenómeno en aspectos como su intensificación y decaimiento, sino además por los potenciales impactos sociales y económicos que puede producir.

Este estudio tiene entre sus objetivos, estudiar la evolución temporal y espacial de las DE asociadas al huracán Otto a su paso desde el Mar Caribe (MC) a través de 
CA hasta el PTE en noviembre de 2016. Este huracán ingresó a tierra en el sureste del territorio de Nicaragua, cerca de la frontera entre este país y Costa Rica, el día 24 de noviembre de ese año. Un dato importante es que Otto es el único huracán con trayectoria más al sur en esta región en toda la historia documentada que atravesó parte de lo que se conoce como el Corredor Seco Centroamericano (CSC), una región con tendencia a las sequías (Hidalgo et al., 2019). Las siguientes secciones de este trabajo tratan sobre los alcances del estudio, los procedimientos empleados, el área de interés (entre ésta, el CSC) y sobre generalidades del clima regional, en especial en el mes de noviembre cuando Otto se desarrolló en el MC. Algunas de las características climáticas del CSC aparecen en esa misma sección. Después de este apartado, se presenta una breve reseña de las principales condiciones sinópticas de la depresión tropical que dio origen a Otto, basada en el trabajo de Brown (2017). La sección siguiente contiene una breve discusión sobre los datos de la WWLLN analizados, la información satelital utilizada y la forma en que se interpreta esta información. Los resultados y las conclusiones se presentan en las secciones finales, junto a algunas consideraciones para orientar el trabajo futuro.

\section{Datos}

La mayoría de DE alrededor del mundo son producidas de nube a nube $(\mathrm{NN})$ con respecto a las descargas nube-tierra (NT) y son producto de cargas eléctricas dentro de las nubes de tormenta (Sadighi, 2015). Estas nubes tienen carga positiva y negativa como la configuración de un tripolo eléctrico (Takahashi, 1978). Entre las plataformas de observación de este tipo de DE está la WWLLN, que es una red de estaciones con sensores que detectan ondas de muy baja frecuencia (VLF, por sus siglas en inglés) de 3 a $30 \mathrm{kHz}$ (Collier et al., 2010) a nivel global en tiempo real, aproximadamente. Los rayos producen ondas VLF, a las cuales la ionosfera es parcialmente permeable de manera que el pulso electromagnético o "sferic" puede viajar, en la guía de onda Tierra-ionosfera, a grandes distancias (Collier et al., 2010).

La WWLLN comenzó su red con 11 estaciones en el 2003, con sensores ubicados en regiones del este de Asia, Australia, Europa, África y América del Norte. Para el 2006, la red contaba con alrededor de 23 estaciones VLF, incorporando nuevas zonas como el subcontinente Indio, el norte de Asia, América del Sur y la Antártida (Rodger et al., 2005; Rodger et al., 2006). Para el año 2008 operaban 27 estaciones de VLF, año en que se incorporó el Centro de Investigaciones Geofísicas (CIGEFI) de la Universidad de Costa Rica $\left(9.94^{\circ} \mathrm{N}, 84.05^{\circ} \mathrm{W}\right)$ a la WWLLN, lo que mejoró la detección de rayos en CA y regiones cercanas. A partir de ese año, la eficiencia en la detección y la ubicación de rayos aumentó debido a nuevas estaciones instaladas en diferentes puntos alrededor del mundo, como había sucedido del 2003 al 2007 con un considerable aumento de sensores y un nuevo algoritmo de procesamiento para la localización de las descargas (Rodger et al., 2009). En la actualidad, operan alrededor de 70 estaciones VLF de la WWLLN.

De acuerdo a Stevenson et al. (2018), la WWLLN es capaz de detectar ambas descargas, tanto NT como NN, aunque la eficiencia de las NT es el doble de la de las NN. En el estudio realizado por Bürgesser (2017), la WWLLN presentó una buena correlación en la distribución espacio temporal de DE con respecto a la Lightning Imaging Sensor (LIS). Además, Rudlosky y Shea 2013 y Thompson et al., 2014, reportan que la WWLLN tiene una mayor capacidad de detección de rayos sobre regiones oceánicas que la LIS. Con estos resultados, la WWLLN genera entonces información apropiada para realizar este trabajo. Los datos satelitales usados para definir las regiones convectivas, en la evolución del huracán Otto 2016, se encuentran en el sitio web CIMSS.

\section{Alcances y Métodos}

Otto tuvo varias etapas durante su desarrollo caracterizadas por una fuerte actividad convectiva (Brown, 2017). El principal periodo de análisis de este trabajo se extiende desde el 23 de noviembre a las 1800 UTC hasta el día 25 de noviembre a las 0300 UTC (en adelante periodo P). Este estudio propone identificar si la intensificación del huracán Otto se refleja en el número de DE y en su distribución espacio-temporal. Otro elemento que se desea investigar es si la distribución espacio-temporal de las DE, antes de ingresar a tierra desde el MC, sufre cambios temporales como ha sido reportado para otros CT (Solórzano et al., 2008). Para esto, se estudia la estructura radial de las $\mathrm{DE}$ en un sistema de coordenadas centradas en $11.10^{\circ} \mathrm{N} \mathrm{y}$ $82.61^{\circ} \mathrm{W}$ para el 24 de noviembre, utilizando tres periodos cada uno de seis horas y la categoría correspondiente de huracán en la escala de Saffir-Simpson (disponible en el sitio web NHC). El primero de estos periodos corresponde de las 0000 UTC - 0500 UTC (huracán categoría 1), el segundo de las 0600 UTC - 1100 UTC (huracán categoría 2) y por último de las 1200 UTC - 1700 UTC (huracán categoría 3 ).

Otro alcance de esta investigación es analizar la distribución espacial y la energía de las DE de Otto en función de la categoría de huracán alcanzada en la escala de Saffir-Simpson. La energía de cada DE fue clasificada como débil, moderada y fuerte, modificando la forma de discriminar las clases usadas por Iwasaki (2015). Para esta clasificación se consideró tanto la energía detectada de la ED (EDE) como también la incertidumbre (IDE), ambas cantidades calculadas y reportadas por la WWLLN. Los resultados están basados en las siguientes consideraciones. Primero que todo, se trabajó solamente con datos de DE 
cuya IDE fuera menor al $50 \%$ de la EDE y diferente de cero. En el primer caso, si la IDE es mayor a la mitad de la $\mathrm{EDE}$, la energía no está en el rango de incertidumbre aceptable y este dato no se toma en cuenta. En este trabajo una $\mathrm{DE}$ débil corresponde a valores menores del percentil 50 de la EDE (971 J), la DE se define moderada cuando la EDE es mayor o igual al percentil 50 de la EDE, pero menor que el percentil $80(3276 \mathrm{~J})$ y la DE se clasifica como fuerte cuando la EDE es mayor o igual al percentil 80.

Un objetivo adicional es investigar si hubo impactos sociales que puedan ser adjudicados a las DE de Otto en la región, en especial en Guanacaste y el suroeste de Nicaragua en el CSC. Estas regiones tienen una densidad poblacional moderada, en especial cerca de las zonas costeras donde el turismo es un factor económico muy importante para el desarrollo de la región. En estas zonas, la pesca deportiva, los paseos en bote y las actividades al aire libre son parte esencial de la economía regional.

\section{EI Clima del Área de Estudio}

La principal área de interés comprende el oeste del Caribe, el Pacífico central del este y Guanacaste de Costa Rica y la región del oeste de Nicaragua (Fig. 1). Varios elementos del clima distinguen estas regiones a pesar de la relativa poca distancia (menos de $200 \mathrm{~km}$ ) que separa esas dos áreas. Por un lado, en el Pacífico se identifican dos máximos de lluvia al año, uno en junio y el otro en septiembre-octubre. En julio-agosto se destaca una reducción importante de precipitación en esta región que ha sido denominada Mid-Summer Drought (MSD, "veranillo" en español; Magaña et al., 1999; Amador, 2008). El MSD forma parte del ciclo anual en el Pacífico, su intensidad y magnitud muestran una variabilidad interanual muy importante que ha sido ligada mediante una correlación negativa significativa con El Niño 3.4 y una relación positiva significativa con un índice de intensidad definido para el CLLJ (Maldonado et al., 2016).

En contraste, la región Caribe muestra una época lluviosa casi continua, con mínimos relativos en mayo y octubre (Sáenz y Amador, 2016). En julio, en esta misma región se presenta un máximo cerca de Barra del Colorado en Costa Rica (ver sitio en Fig. 1) que ha sido vinculado con la convergencia de flujo del CLLJ (Amador, 1998). En el trabajo de Hidalgo et al. (2015) se ofrece una visión actualizada de la relación entre el CLLJ, el desplazamiento de la ZCIT y la distribución de lluvias en la región de Centroamérica, incluyendo el área de interés de esta investigación. A lo largo del Pacífico de CA y sur de México existe un área denominada el CSC que favorece el desarrollo de ecosistemas de bosque seco con una definida y seca estación (Calvo et al., 2018) que incluye el MSD. El CSC es además una región que sufre el impacto de El Niño con anomalías positivas de temperatura superficial

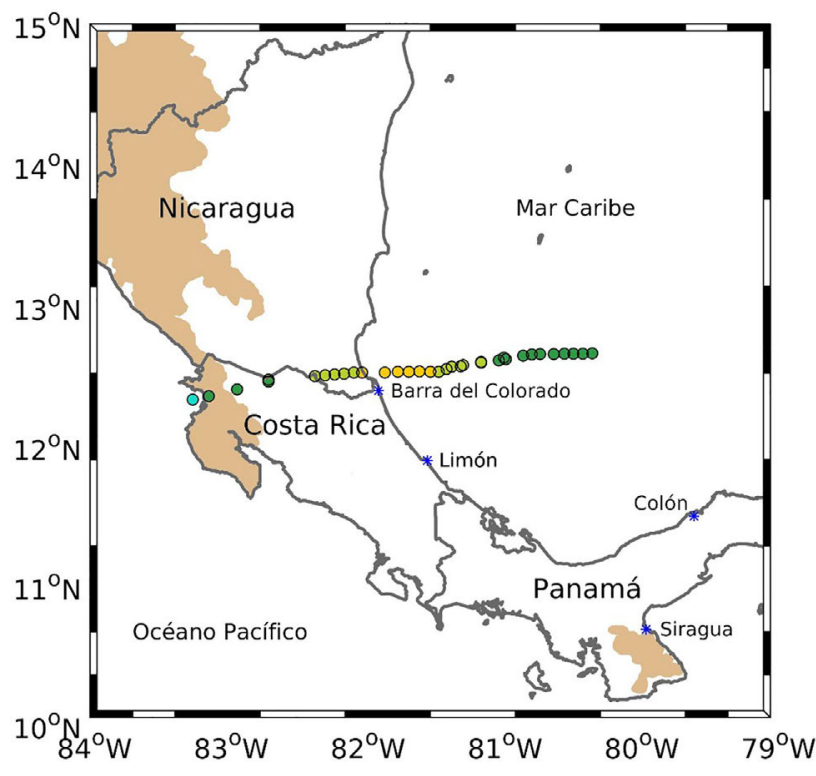

Figura 1 - Trayectoria del huracán Otto durante el periodo 23 de Noviembre (2300 UTC) al 25 de noviembre (0300 UTC) de 2016, mostrando su condición como huracán categoría 1 (círculos verde oscuro); huracán categoría 2 (círculos verde claro); huracán categoría 3 (círculos amarillos) antes de su ingreso a tierra y su degradación a huracán categorías 2 y 1, a medida que cruza Nicaragua y Costa Rica, hasta su condición de tormenta tropical (círculo turquesa) al ingresar al Pacífico tropical del este (Brown 2017). La escala de huracanes utilizada es la de Saffir-Simpson. El área que se muestra es la región de análisis de la actividad eléctrica asociada a Otto, mientras que el área sombreada en café claro representa el Corredor Seco Centroamericano CSC aproximadamente (adaptada de CCAD 2011).

del orden de $1{ }^{\circ} \mathrm{C}$ o más durante esta fase (Alfaro y Amador, 1996, 1997), al tiempo que el gradiente de presión Caribe-Pacífico es mayor a lo normal, los vientos alisios son más fuertes durante la fase cálida del ENOS durante los veranos boreales (Amador y Alfaro, 1996; Amador et al., 2003; Amador 2008; Amador et al., 2010). Una de las características del CSC es la frecuencia de períodos secos durante la estación lluviosa (Peralta Rodríguez et al., 2012) que afecta la ecología de esta región. Hidalgo et al. (2019) reportan que a pesar de que el estrés hídrico en CA es bajo, el CSC es una región sujeta a sequías casidecenales relacionadas con la intensificación del CLLJ, de ahí la importancia de analizar casos de huracanes como el de Otto que generan abundante precipitación en esa zona en épocas de poca lluvia (noviembre). A pesar de la migración latitudinal de la ZCIT en la región de CA, hay otras zonas que presentan deficiencias de lluvia apreciables, tal es el caso del Parque Nacional Siragua (Fig. 1), una zona árida de cerca de 8000 hectáreas en la provincia de Herrera en Panamá. A pesar de la afluencia a la región de muchos sistemas meteorológicos, entre ellos, las ondas ecuatoriales y los CT (y sus efectos directos o indirectos; Peña y Douglas, 2002) las regiones mencionadas como el CSC y el Sarigua mantienen un balance hídrico muchas veces deficitario. En este sentido la presencia de un $\mathrm{CT}$ 
como Otto en el 2016 sobre el CSC y el Sarigua ayuda a disminuir en alguna medida el estrés hídrico anual en estas zonas.

La región de estudio presenta una intensa actividad ciclónica tanto a nivel estacional como interanual. Amador et al. (2016a, b) muestran que para el periodo 1966-2014 predomina una alta variabilidad del número de $\mathrm{CT}$ año a año tanto para el PTE como para la cuenca del MC. Para el PTE, el número total de tormentas tropicales (TT), el número de huracanes categoría 1 y 2 (escala de SaffirSimpson) ó el número de huracanes mayores (categoría 3 a 5) no mostraron ninguna tendencia significativa al $99 \%$ (de acuerdo con la prueba de Mann-Kendall) para el periodo usado. En el MC, sólo el número de TT mostró una tendencia positiva significativa al $99 \%$ para el mismo periodo. En estos trabajos se documenta que, aunque los máximos de frecuencia estacional de estos sistemas coinciden para las dos cuencas en septiembre, en cuanto al número de TT y huracanes mayores, la actividad disminuye notoriamente en el Pacífico en octubre, mientras que en el MC esta actividad persiste de manera clara hasta noviembre, mes en que Otto se desarrolló en el 2016.

\section{Reseña Sinóptica del Huracán Otto}

Esta sección presenta un resumen general de Otto, siguiendo a Brown (2017), con el interés de ubicar geográficamente este trabajo desde la perspectiva sinóptica. El sistema se originó como una depresión tropical en el suroeste del Caribe el día 15 de noviembre del 2016. A partir de entonces y hasta el 20 de noviembre se observó una circulación poco organizada con fluctuante actividad convectiva y anomalías en la velocidad de los vientos. Una alta presión sobre el Golfo de México y dos empujes fríos que incursionaron hasta el MC intensificaron la formación de Otto. El 20 de noviembre a las 1800 UTC fue declarado depresión tropical al norte de Colón, Panamá (la Fig. 1 muestra la localización de Colón). Durante el 21 de noviembre, Otto se convirtió en una TT con vientos de $120 \mathrm{~km} / \mathrm{h}$ y velocidad de traslación de $4 \mathrm{~km} / \mathrm{h}$ con $984 \mathrm{hPa}$ en su centro y fue ubicado aproximadamente a $975 \mathrm{~km}$ al este de Puerto Limón, Costa Rica (ver la localización de Limón en la Fig. 1). Otto alcanzó categoría de huracán 1 en la escala de Saffir-Simpson (en adelante OH1) el día 23 de noviembre (1800 UTC) cuando estaba localizado aproximadamente a unos $250 \mathrm{~km}$ al noreste de Limón. Alrededor de las 0943 UTC del 24 de noviembre se logró observar el ojo del huracán con una categoría $2(\mathrm{OH} 2)$, ubicado a $115 \mathrm{~km}$ al norte de Limón. Otto con una categoría $3(\mathrm{OH} 3)$ logró impactar la Reserva Biológica Indio Maíz ubicada al sureste de Nicaragua en el Caribe (al norte de Barra del Colorado en Costa Rica) a las 1730 UTC del día 24 de noviembre (ver las diferentes posiciones del huracán y su categoría en la Fig. 1). Luego de atravesar parte del CSC entre Costa Rica y Nicaragua se debilitó, convirtiéndose en TT antes de salir al Océano Pacífico, el día 25 de noviembre cerca de las 0330 UTC. La Fig. 1 muestra la trayectoria de Otto adaptada del sitio web RAMMB CIRA con las categorías que alcanzó este huracán en colores de acuerdo a NESDIS.

Como antecedentes de Otto en el MC y CA, se pueden mencionar dos huracanes históricos que impactaron de manera fuerte a la región, cuyas trayectorias fueron algo similar a las de Otto, solo que un poco más al norte de Costa Rica. Se trata de Joan en octubre de 1988, que tocó tierra el 22 de ese mes cerca de Bluefields en Nicaragua, cruzó CA como huracán categoría 5 y continuó su trayectoria como Miriam en el Pacífico. El otro caso es el de Cesar en julio de 1996 que también tocó tierra cerca de Bluefields y que como TT pasó al Pacífico donde adquirió el nombre de Douglas. Información sobre la trayectoria, otras características de Joan y Cesar, así como los principales impactos de estos huracanes se detallan en Lawrence and Gross (1989) y Pasch and Avila (1999), respectivamente. En ninguno de los casos anteriores se incorporó un análisis de la actividad eléctrica de los $\mathrm{CT}$, por lo que el presente trabajo, aunque inicial, es pionero en ese sentido en CA.

\section{Resultados}

La Fig. 2 muestra la distribución temporal de las diferentes fases de actividad eléctrica de Otto durante el periodo P. Algunas etapas de este periodo se caracterizaron por una rápida intensificación de la actividad eléctrica de Otto. Se pueden notar los tres picos de máxima actividad de DE para todo el periodo convectivo de Otto como OH1 (23 de noviembre 1800 UTC al 24 de noviembre 0500 UTC circa, barras verdes oscuro), la fase de intensificación del ojo del huracán en $\mathrm{OH} 2$ (24 de noviembre de las 0600 UTC a las 1100 UTC circa, barras verdes claro) y el incremento de esta actividad antes de la entrada de Otto a tierra en la fase $\mathrm{OH} 3$ ( 24 de noviembre de las 1200 UTC a las 1700 UTC circa, barras amarillas). A partir de esta fase las DE disminuyeron debido a la degradación de Otto tras su ingreso a tierra hasta salir al Pacífico costarricense como TT (barra turquesa). La línea de puntos y círculos rojos es el acumulado de las descargas durante el periodo $\mathrm{P}$ con un total de casi 35000 rayos en 34 horas. La línea negra a rayas representa el promedio de descargas para todo el periodo estudiado (un poco más de 1000 descargas por hora).

Es a partir del 23 de noviembre a las 2300 UTC que este sistema presenta cerca del centro de rotación (Fig. 3a), una fuerte actividad eléctrica ( $\mathrm{OH} 1)$. Después de esto, Otto mostró una disminución apreciable de este proceso entre las 0300 UTC y 0400 UTC del 24 de noviembre (Fig. 3b) para luego alcanzar el segundo máximo de actividad entre las 0500 UTC y las 0900 UTC del mismo día. El pico máximo de este ciclo se observó a las 0700 UTC (Fig. 3c) con bandas muy activas al norte del centro del 


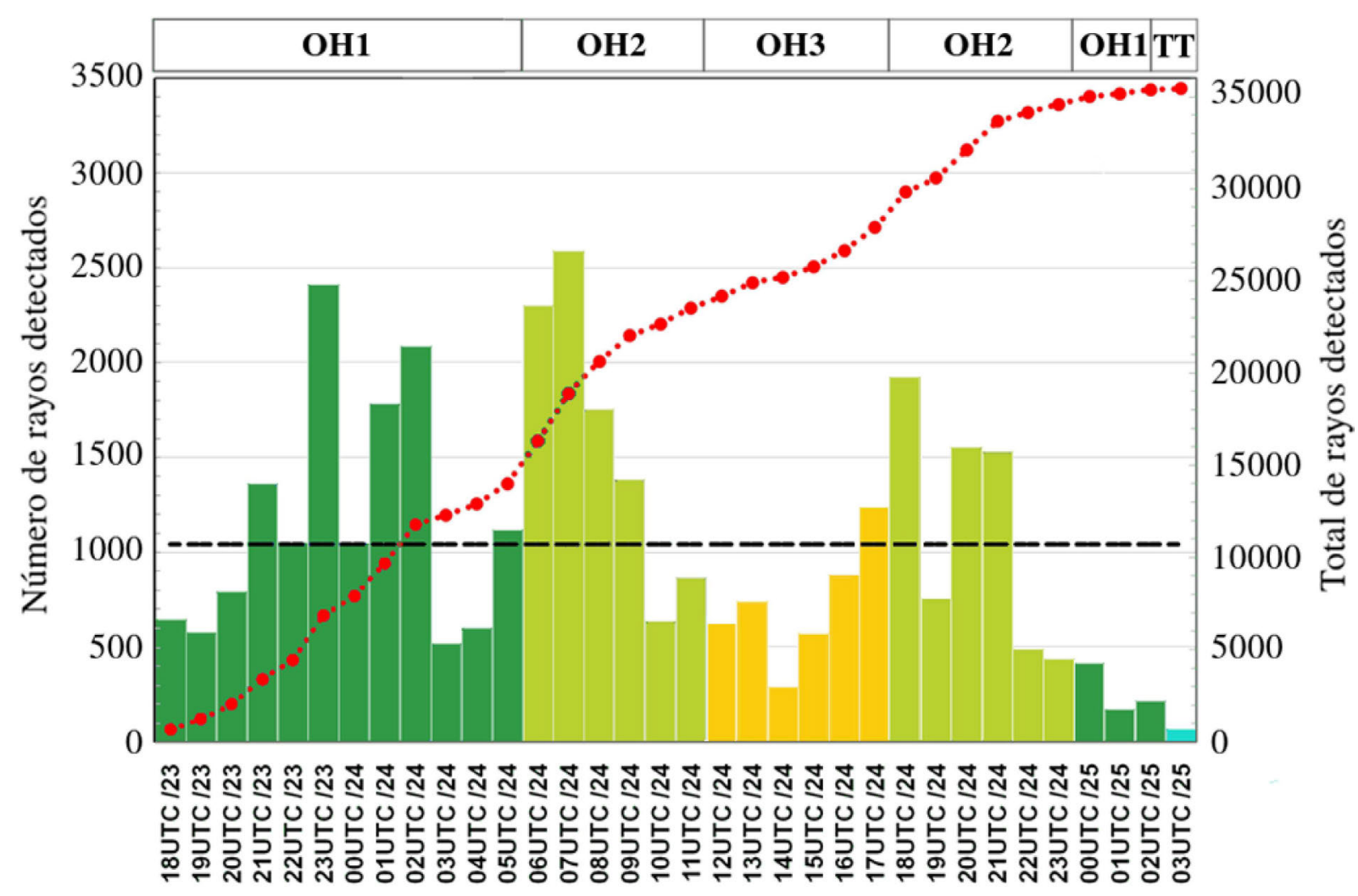

Figura 2 - Distribución temporal de las diferentes fases de actividad eléctrica de Otto durante el periodo 23 noviembre (2300 UTC) al 25 de noviembre (0300 UTC) 2016. Nótense los tres picos máximos de actividad de descargas eléctricas para el periodo convectivo inicial (huracán categoría 1, OH1, barras verdes oscuro), la fase de intensificación del ojo del Otto (huracán categoría 2, OH2, barras verdes claro) y el incremento moderado de esta actividad antes de la entrada de Otto a tierra (categoría 3, OH3, barras amarillas). A partir de esto Otto se degradó hasta salir al Pacífico costarricense como tormenta tropical (barra turquesa) y las DE disminuyeron notablemente. La línea de puntos y círculos rojos es el acumulado de las descargas durante este periodo con un total de casi 35000 rayos en 34 horas. La línea negra a rayos representa el promedio de descargas para todo el periodo (un poco más de 1000 descargas por hora). Datos de la red WWLLN.

huracán, región que evidenció una disminución apreciable de $\mathrm{DE}(\mathrm{OH} 2)$. Un periodo de menor actividad se presentó desde las 1000 UTC hasta las 1600 UTC de ese día. Esta fase se caracterizó por una desorganización de las bandas de actividad eléctrica a pesar de que la convección debida a Otto era notablemente visible (disponible en el sitio web CIMSS). La Fig. 3d muestra para las 1400 UTC de ese día una notable disminución en la actividad eléctrica especialmente cerca del ojo del huracán, lo que concuerda con los trabajos de Molinari et al. (1994) y DeMaria et al. (2011).

Los paneles a, b y c de la Fig. 4 muestran claramente como antes y poco después de su ingreso a tierra, la convección y la actividad eléctrica se vigorizan sobre el océano mostrando una notable asimetría en su distribución con respecto a tierra. De acuerdo a Abarca et al. (2011) esta asimetría podría deberse a la velocidad de desplazamiento y a la cortante del viento asociada a Otto, aun cuando estas relaciones no están del todo claras. En regiones cercanas al ojo del huracán se nota una relevante disminución de la $\mathrm{DE}$, lo que concuerda con lo discutido por Solórzano et al. (2008). Una vez sobre Nicaragua,
Otto pierde intensidad al disminuir su fuente de evaporación y aumentar la fricción superficial; casi al tiempo que se observa una notoria disminución en la actividad eléctrica (Fig. 4d).

En la Fig. 5 se muestran imágenes de satélite de la temperatura del tope de la nube (paneles superiores) y la superposición de estas mismas imágenes con las DE para a) 2300 UTC del día 23, b) 0300 UTC del día 24, c) 0700 UTC del día 24 y d) 1400 UTC del día 24, en noviembre de 2016. En la Fig. 5a, la mayor parte de las DE se asocian a las bandas de convección cerca de la pared del ojo $\mathrm{OH} 1$ con presencia de temperaturas muy bajas en el tope de la nube (alrededor de los $-80^{\circ} \mathrm{C}$ y $-90^{\circ} \mathrm{C}$ ); caso contrario con la hora 0300 UTC (Fig. 5b) que se observaron temperaturas muy bajas (zonas de color negro y rosado) pero una disminución de las DE. En la Fig. 5c (OH2) se aprecian varias bandas externas muy bien definidas con una alta actividad eléctrica que corresponden a regiones de intensa convección y topes de nubes con temperaturas entre $-70{ }^{\circ} \mathrm{C} \mathrm{a}-80{ }^{\circ} \mathrm{C}$. Para la hora 1400 UTC, tres horas antes del ingreso a tierra (OH3), se observa el ojo del huracán con un mínimo en las DE y aumento relativo de la 
temperatura en los topes de las nubes entre $-60{ }^{\circ} \mathrm{C}$ a $-70{ }^{\circ} \mathrm{C}$ (zonas de color roja principalmente).

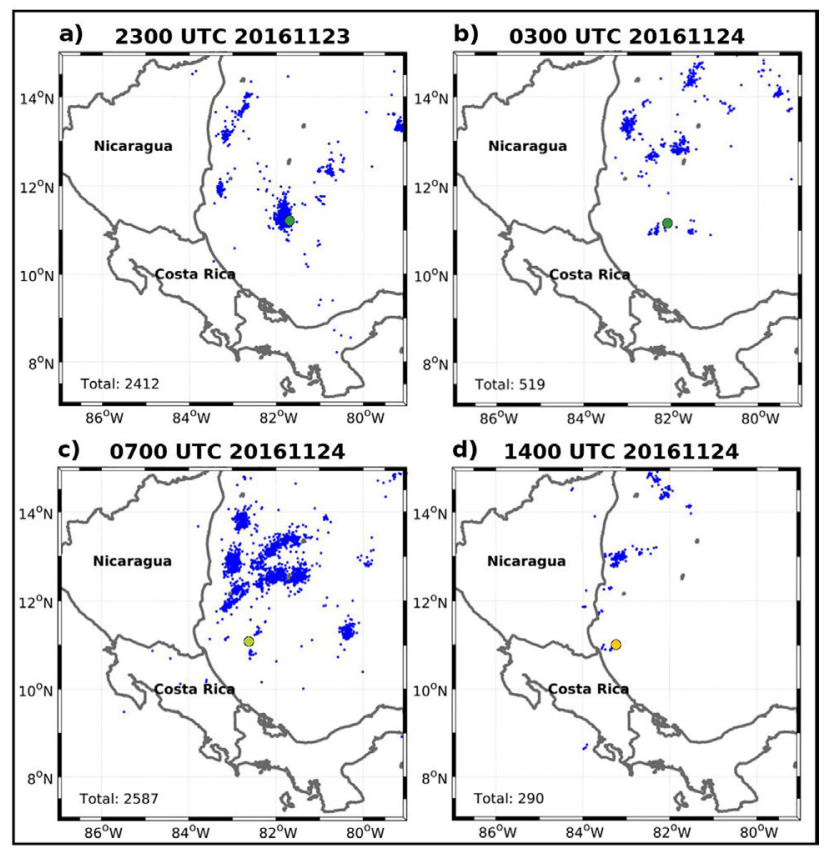

Figura 3 - Distribución espacial de descargas eléctricas (DE) para el huracán Otto durante el periodo 23 Noviembre (2300 UTC) hasta el 24 de noviembre (2200 UTC) 2016, con respecto a la posición del centro del huracán según categoría en la escala de Saffir-Simpson, para a) el primer pico de descargas eléctricas del periodo analizado con $2412 \mathrm{DE}$; b) primer mínimo de actividad de descargas (519 DE); c) máximo absoluto con bandas de intensa actividad eléctrica durante la intensificación del ojo del huracán con $2587 \mathrm{DE}$; d) mínimo absoluto de $290 \mathrm{DE}$ poco antes de que Otto entrara a tierra entre Costa Rica y Nicaragua. Datos de la red WWLLN
En cuanto a la distribución espacial de las descargas, Molinari et al. (1999) examinaron las NT para nueve huracanes en el Atlántico usando datos de la National Light-

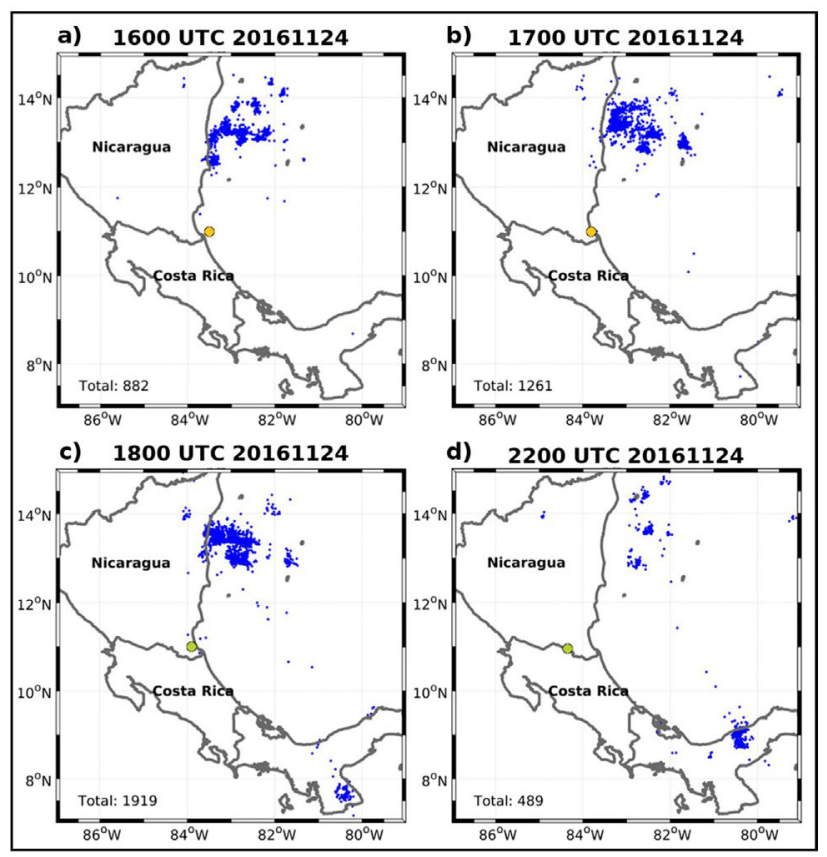

Figura 4 - Distribución espacial de descargas eléctricas (DE) para el huracán Otto durante el periodo 23 Noviembre (2300 UTC) hasta el 24 de noviembre (2200 UTC) 2016, con respecto a la posición del centro del huracán según categoría en la escala de Saffir-Simpson, para a) intensificación de Otto mostrando algunas bandas con intensa actividad eléctrica antes de su entrada a tierra entre Costa Rica y Nicaragua (882 DE); b) incremento de la actividad eléctrica al ingresar a tierra (1261 DE); c) como en b) pero con un máximo de $1919 \mathrm{DE}$ y d) des-intensificación de Otto sobre el norte de Costa Rica con 489 DE. Datos de la red WWLLN.
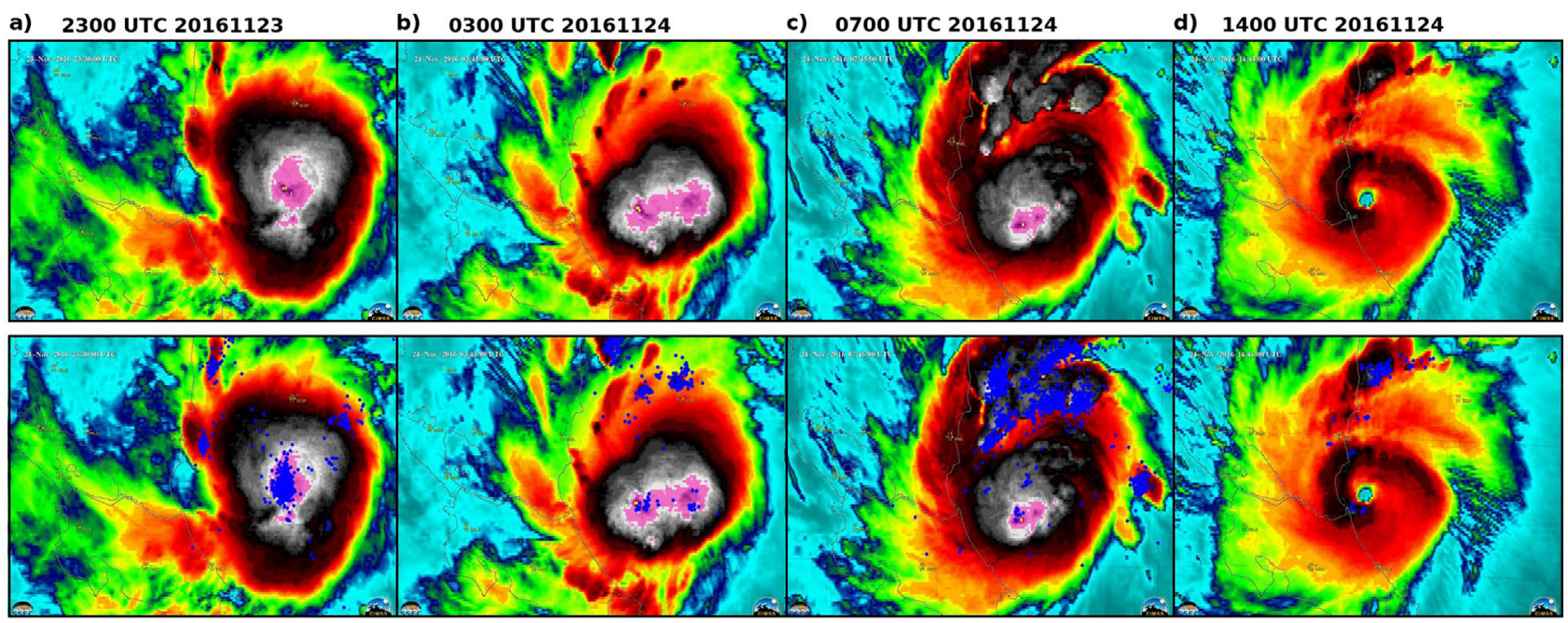

$\begin{array}{llllllll}-20 & -30 & -40 & -50 & -60 & -70 & -80 & -900^{\circ} \mathrm{C}\end{array}$

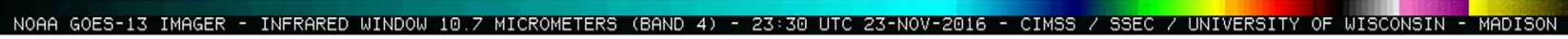

Figura 5 - Temperatura del tope de la nube y las descargas eléctricas del huracán Otto para las horas: a) 2300 UTC del día 23, b) 0300 UTC del día 24, c) 0700 UTC del día 24 y d) 1400 UTC del día 24. Datos de satélite de CIMSS y de la red WWLLN. 
ning Detection Network (NLDN por sus siglas en inglés) y encontraron que la actividad eléctrica a $40 \mathrm{~km}$ del centro del huracán para cuatro de los huracanes estudiados con categoría de moderados a fuertes ocurrió esporádicamente. La distribución radial de descargas presentó un máximo débil en la región de la pared del ojo del huracán, un mínimo fuera de esta región entre 80-100 km y un fuerte máximo entre 200 y $300 \mathrm{~km}$ circa. Estos autores reportan que las bandas externas de convección con valores medios máximos de densidad de rayos de la NLDN cerca de los $250 \mathrm{~km}$ contenían la mayor parte de los rayos NT. Basados en el trabajo anterior Cecil et al. (2002) categorizaron los perfiles radiales de $\mathrm{DE}$ en tres secciones; la región de la pared del ojo, las regiones internas y externas debidas a las bandas de lluvia. En términos generales, la distribución de perfiles radiales de Molinari et al. (1999) corresponde a las regiones propuestas por Cecil et al. (2002). En este trabajo no se hace una categorización previa de regiones como en los dos trabajos anteriores, sino que se generan perfiles radiales desde el centro del huracán hacia afuera cada $50 \mathrm{~km}$ hasta los $550 \mathrm{~km}$ con el objetivo de estudiar las DE en función del radio y de la categoría del huracán según se desprende de la Fig. 1. En la Fig. 6 se observa que para radios menores a $50 \mathrm{~km}$ (región de la pared del ojo aproximadamente) solo en el caso de $\mathrm{OH} 1$ hubo un número alto de descargas que corresponde al $34 \%$ de todas las ocurridas durante esa categoría de huracán. La región interna del huracán con radios menores a $100 \mathrm{~km}$ no presenta gran actividad eléctrica para ninguna de las tres categorías de huracán alcanzadas por Otto. La mayor actividad eléctrica para $\mathrm{OH} 2$ se encuentra entre los 100 y $300 \mathrm{~km}$ (región de bandas de lluvias externas), en acuerdo con los resultados de Molinari et al. $(1994,1999)$ y Cecil et al. (2002). Fuera

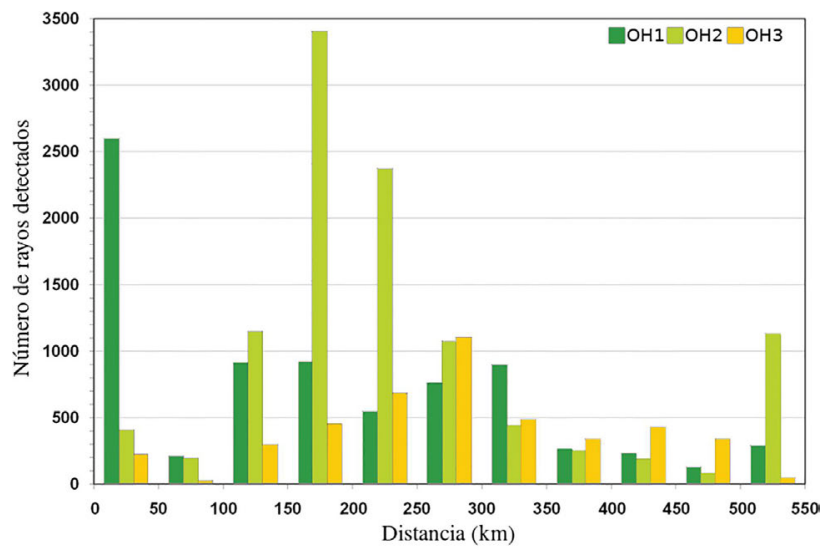

Figura 6 - Frecuencia relativa de la distribución de las descargas eléctrica del huracán Otto en un sistema de coordenadas centradas en $11.1^{\circ} \mathrm{N}, 82.6^{\circ} \mathrm{W}$ a una distancia de $50 \mathrm{~km}$ desde el centro del huracán hasta los $550 \mathrm{~km}$, durante el 24 de noviembre del 2016 para el periodo de 0000 UTC -0500 UTC (categoría 1, barras verdes oscuro), 0600 UTC 1100 UTC (categoría 2, barras verdes claro) y 1200 UTC - 1700 UTC (categoría 3, barras amarillas). La escala de huracanes utilizada es la de Saffir-Simpson como en la Fig. 1. Datos de la red WWLLN. de esta región circular de $300 \mathrm{~km}$ las descargas disminuyen notablemente. El periodo con mayor actividad eléctrica dentro de los $500 \mathrm{~km}$ corresponde al $\mathrm{OH} 2$ (un total de $10638 \mathrm{DE}$ ) y el de menor actividad al OH3 (un total de $4385 \mathrm{DE}$ ) que tiene su máximo entre los 250 y $300 \mathrm{~km}$. El huracán Otto presentó una asimetría notable en sus bandas de convección desde su inicio como OH1; los cuadrantes IV y I (Fig. 7) presentan la mayor cantidad de DE en todas las fases de intensificación desde $\mathrm{OH} 1$ a $\mathrm{OH} 3$. Con respecto al cuadrante I, la frecuencia relativa para $\mathrm{OH} 3$ fue del $80 \%$, OH2 de $56 \%$ y OH1 $46 \%$, al contrario del cuadrante II con frecuencias relativas muy bajas de $2 \%, 1 \%$ y $9 \%$ respectivamente.

El análisis de la distribución espacial y la energía de las DE para el huracán Otto en un sistema de coordenadas centrado en $11.1^{\circ} \mathrm{N}, 82.6^{\circ} \mathrm{W}$ para los tres periodos $(\mathrm{OH} 1$, $\mathrm{OH} 2$ y OH3) se presenta en la Fig. 8. Las DE en color azul que no tienen un rango de incertidumbre aceptable, corresponden a un $87.8 \%$ para $\mathrm{OH} 1$, a $83.5 \%$ para $\mathrm{OH} 2$ y a $81.7 \%$ para $\mathrm{OH} 3$, lo cual refleja un aspecto a mejorar en la WWLLN para un futuro. En las tres categorías de Otto la mayor parte de DE que cumplieron con las condiciones establecidas (IDE mayor a cero y que no supera el $50 \%$ de la EDE) fueron categorizadas como débiles (para $\mathrm{OH} 1$, $7.3 \%$; $\mathrm{OH} 2,9.2 \%$ y OH $3,11.5 \%$ ) y la menor parte de estas corresponden a DE fuertes (para OH1, 1.7\%; OH2, 2.9\% y $\mathrm{OH} 3,2.4 \%$ ). En la Fig. 9 se puede también observar que la red de rayos WWLLN durante el periodo $\mathrm{P}$ tuvo una mayor detección de $\mathrm{DE}$ con energías bajas y una menor detección de DE con energías altas.

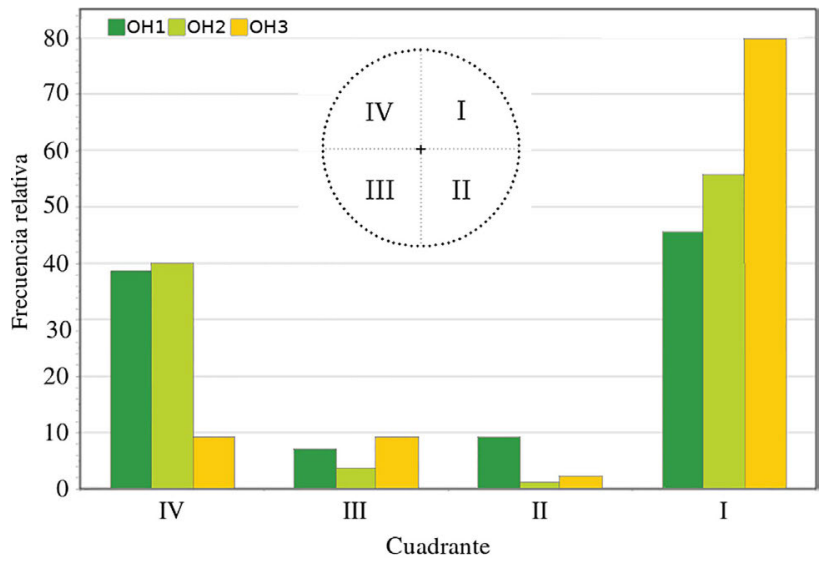

Figura 7 - Frecuencia relativa de la distribución de las descargas eléctrica $(\mathrm{DE})$ por cuadrantes meteorológicos $\left(0^{\circ}\right.$ corresponde al Norte, $90^{\circ}$ al Este, $180^{\circ}$ al Sur y $270^{\circ}$ al Oeste) del huracán Otto en un sistema de coordenadas centradas en $11.1^{\circ} \mathrm{N}, 82.6^{\circ} \mathrm{W}$ durante el 24 de noviembre del 2016 para el periodo de 0000 UTC - 0500 UTC (categoría 1, barras verdes oscuro), 0600 UTC - 1100 UTC (categoría 2, barras verdes claro) y 1200 UTC - 1700 UTC (categoría 3, barras amarillas). Se observa una asimetría en la distribución de las DE, en el cuadrante II se muestra mayor DE para las tres categorías del huracán; además menor DE en el cuadrante III y IV. Para el huracán categoría 3, hay una disminución de DE en el cuadrante III comparado al huracán categoría 1 y 2 . La escala de huracanes utilizada es la misma de la Fig. 1. Datos de la red WWLLN. 


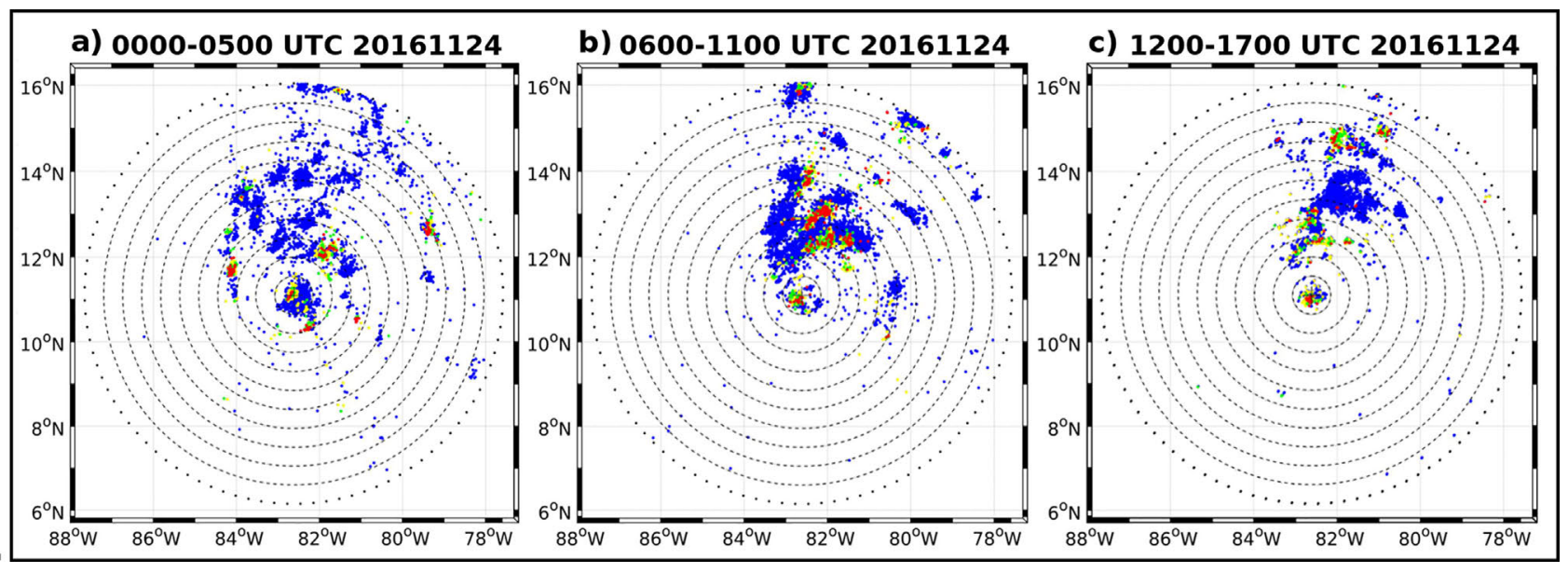

Figura 8 - Distribución espacial de descargas eléctricas (DE) para el huracán Otto en un sistema de coordenadas centradas en $11.1^{\circ} \mathrm{N}, 82.6^{\circ} \mathrm{W}$ durante el 24 de noviembre del 2016 para los periodos de: a) 0000 UTC - 0500 UTC (OH1), b) 0600 UTC - 1100 UTC (OH2) y c) 1200 UTC - 1700 UTC (OH3), además muestra la energía asociada a las DE de cada periodo para diferentes categorías: débil (puntos verdes), moderados (puntos amarillos) y fuertes (puntos rojos). La escala de huracanes utilizada es la de Saffir-Simpson como en la Fig. 1. Datos de la red WWLLN.

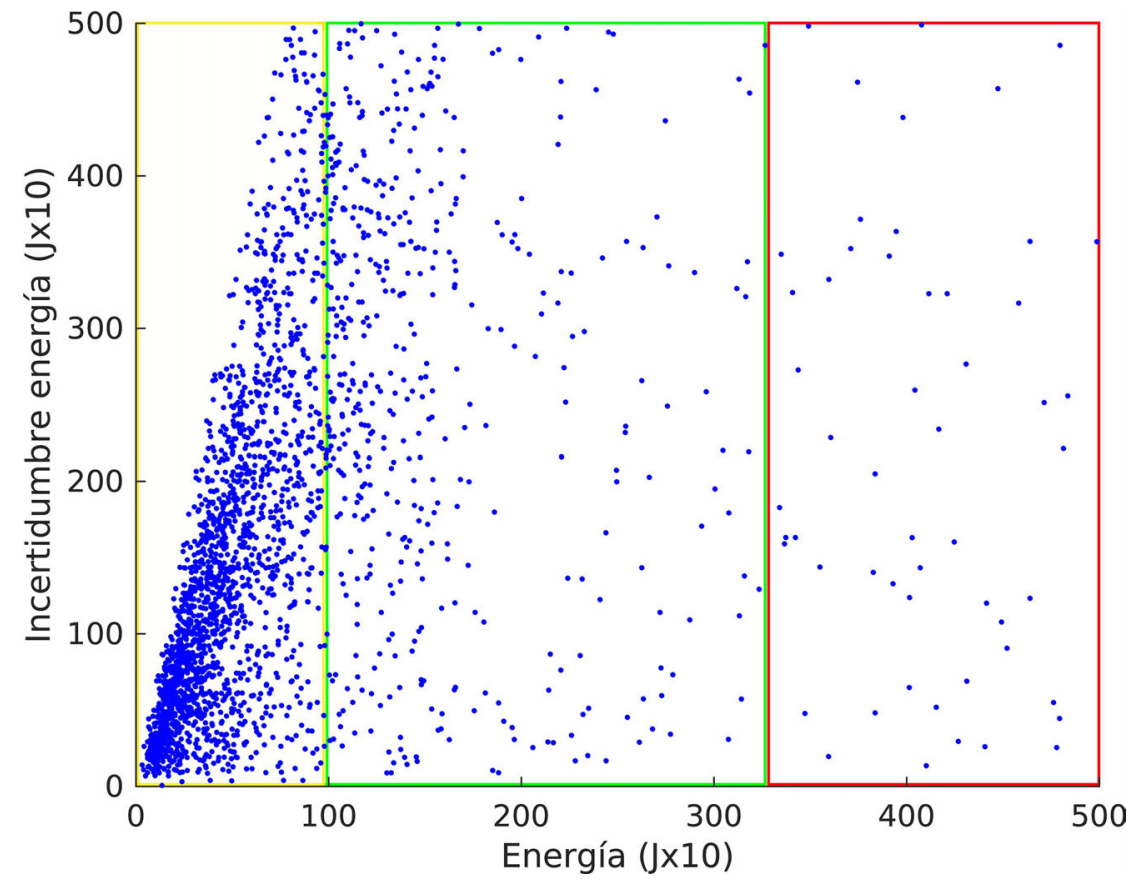

Figura 9 - Gráfico de dispersión para la incertidumbre asociada a la energía de las descargas eléctricas para el periodo de estudio 0000 UTC- 1700 UTC del 24 de noviembre del 2016. El cuadro amarrillo encierra las descargas eléctricas débiles, cuadro verde las descargas eléctricas moderadas y en rojo las descargas eléctricas fuertes.

De acuerdo a Desastres (2017), Otto afectó a más de diez mil personas en Costa Rica (diez personas fallecidas) y a más de cuatro mil en Nicaragua (no se registraron personas que perdieran la vida en este país), con alrededor de mil seiscientas y ochocientas viviendas afectadas respectivamente. La atenuación en la intensidad de Otto, la disminución en la actividad eléctrica al atravesar Costa Rica y los constantes avisos de previsión emanados de fuentes oficiales de los países, fueron factores importantes para que no se registraran impactos directos de rayos en la población que estaba en condiciones de alta vulnerabilidad por ráfagas de viento $\mathrm{y}$ copiosas lluvias (IMN 2017).

\section{Comentarios Finales}

En este trabajo se analizan las DE del huracán Otto en noviembre del 2016 a su paso por el Caribe y el CSC durante todas sus fases como $\mathrm{OH} 1, \mathrm{OH} 2$ y $\mathrm{OH} 3$ usando datos de la red WWLLN, lo cual constituye una investiga- 
ción pionera en esta región. Las DE asociadas a Otto capturan muy bien las fases de intensificación y degradación de este huracán, haciendo de este parámetro una variable potencial importante en su evolución que sería útil parametrizar en los modelos numéricos para que aporte información en su proceso de evolución. Este resultado es consistente con trabajos anteriores como el de Abarca et al. (2011) y Molinari et al. (1994). De acuerdo a Wong et al. (2013) la mayor parte de los estudios en que las descargas eléctricas han sido parametrizadas han seguido el método propuesto por Price \& Ride (1994) que está basado fundamentalmente en la altura del tope de las nubes. En este trabajo se encontró que la mayor densidad de rayos y la evolución de este parámetro en el huracán Otto está asociado a las temperaturas más bajas del tope de las nubes y no a la altura de esos topes. La temperatura del tope de las nubes no es el único parámetro importante en el proceso de parametrización ya que el contenido total de hielo, la velocidad vertical máxima y la energía potencial convectiva disponible son también elementos básicos del proceso de generación de descargas eléctricas. (Gianaros et al., 2015).

El número de DE observado durante el periodo de análisis corresponde a unos 35000 rayos, lo que significa unos 1000 rayos por hora durante estas etapas como huracán. Durante las fases $\mathrm{OH} 1, \mathrm{OH} 2$ y $\mathrm{OH} 3$, cerca del ojo del huracán (50 km o menos) el número de DE disminuyó notablemente a medida que el huracán se intensificaba. Esto concuerda con lo observado en las imágenes de satélite que indican un movimiento radial de las DE hacia afuera del centro de Otto a medida que cambia de $\mathrm{OH} 1$ a $\mathrm{OH} 3$. En la mayor parte de los casos los núcleos de $\mathrm{DE}$ estaban asociados a la temperatura del tope de las nubes menores a los $-70^{\circ} \mathrm{C}$. La estructura radial de las DE para el huracán Otto muestra una clara relación con la categoría en la escala de Saffir-Simpson que alcanzó este huracán durante las horas 0000 UTC hasta las 1700 UTC del día 24 de noviembre; esto se debe a que la región de mayor cantidad de las DE se desplazó del centro hacia las zonas más externas del huracán conforme este se intensificó. En el caso de OH3, el máximo de DE se ubicó en las bandas externas de Otto $(250-300 \mathrm{~km})$; este resultado concuerda con lo obtenido por Molinari et al. (1994) en su estudio de DE en el huracán Andrew (1992). Otto se caracterizó por una asimetría notable en la distribución de las DE (Fig. 7), siendo esta distribución máxima para los cuadrantes al norte del centro del huracán. La red de rayos WWLLN muestra deficiencias importantes en la detección de las energías DE, ya que más del $80 \%$ en todas las categorías del huracán las energías tenían incertidumbres no utilizables para propósitos de investigación. Aun así, la red tuvo una mayor detección de DE con energías bajas y una menor detección de DE con energías altas para todo el periodo analizado.

\section{Agradecimientos}

Los autores agradecen el apoyo de los siguientes proyectos, VI-805-B8-068/B7-507 (IP Dra. A.M. Durán), B8604 (IP, Dr. E. Rivera), B6-143/B8-766 (IP, Dr. H. Hidalgo), B9-454 (IP, Dr. J. Amador) y B9-609 (IP, Dr. R. Castillo). A Paula Pérez por su colaboración en la construcción de la Fig. 1, al CIGEFI y la Escuela de Física de la Universidad de Costa Rica por las facilidades para realizar esta investigación.

\section{Referencias}

ABARCA, S.F.; CORBOSIERO, K.L.; VOLLARO, D. The World Wide Lightning Location Network and Convective Activity in Tropical Cyclones. Monthly Weather Review, v. 139, n. 1, p. 175-191, 2010.

ABERSON, S.D.; BLACK, M.L.; BLACK, R.A.; BURPEE, R.W.; CIONE, J.J.; LANDSEA, C.W.; MARKS, F.D. Thirty years of tropical cyclone research with the noaa $\mathrm{p}-3$ aircraft. Bulletin of the American Meteorological Society, v. 87, n. 8, p. 1039-1056, 2006.

ALBRECHT, R.; GOODMAN, S.; BUECHLER, D.; BLAKESLEE, R.; CHRISTIAN, H. Where are the lightning hotspots on Earth? Bulletin of the American Meteorological Society, v. 97, p. 2051-2068, 2016.

ALFARO, E.J.; AMADOR, J.A. Variabilidad y cambio climático en algunos parámetros sobre Costa Rica y su relación con fenómenos de escala sinóptica y planetaria. Revista Tópicos Meteorológicos y Oceanográficos, v. 4, p. 51-62, 1997.

ALFARO MARTÍNEZ, E.J.; AMADOR ASTÚA, J.A. El Niñooscilación del Sur y algunas series de temperaturas máxima y brillo solar en Costa Rica. Revista Tópicos Meteorológicos y Oceanográficos, v. 3, n. 1, p. 19-26, 1996 a.

ALFARO MARTÍNEZ, E.J.; AMADOR ASTÚA, J.A. La oscilación cuasi-bienal, ENOS y acoplamiento de algunos parámetros superficiales y estratosféricos sobre Costa Rica. Revista Tópicos Meteorológicos y Oceanográficos, v. 3, n. 1, p. 45-53, 1996b.

AMADOR, J.A. A climatic feature of the tropical Americas: The trade wind easterly jet. Revista Tópicos Meteorológicos y Oceanográficos, v. 5, n. 2, p. 1-13, 1998.

AMADOR, J.A. The Intra-Americas Sea Low-level Jet. Annals of the New York Academy of Sciences, v. 1146, p. 15388, 2009.

AMADOR, J.A. Socio-economic impacts associated with meteorological systems and tropical cyclones in Central América in 2010. Bulletin of the American Meteorological Society, v. 92, n. 6, p. 184, 2011.

AMADOR, J.A.; CHACÓN, R.E.; LAPORTE, S. Climate and climate variability in the arenal river basin of Costa Rica. In: BENISTON, M.; DIAZ, H.F.; MOREHOUSE, B.J. (ed). Climate and Water, v 16. p. 317-349, 2003.

AMADOR, J.A.; CHACÓN, R.E.; LIZANO, O.G. Estudio de efectos geofísicos del Terremoto de Limón mediante percepción remota y análisis hidrometereológico. Revista 
Geológica de América Central, v. esp. Terremoto de Limón, p. 153-170, 1994.

AMADOR, J. A.; ALFARO, E. J.; RIVERA, E. R.; CALDERÓN, B. Climatic Features and their relationship with tropical cyclones over the intra-Americas seas. In: ELSNER, J. B.; HODGES, R. E.; MALMSTADT, J. C.; SCHEITLIN, K. N. (Ed.). Hurricanes and Climate Change, 2010. v. 2, p. 149-173.

AMADOR, J.; HIDALGO, H.; ALFARO, E.; CALDERÓN, B.; MORA, N. AMADOR, J. Central America [in "State of the Climate in 2018"]. Bulletin of the American Meteorological Society, v. 100, n. 9, p. S197-S198, 2019.

AMADOR, J.A.; HIDALGO, H.; ALFARO, E.J.; DURÁNQUESADA, A.M.; CALDERÓN, B.; MORA, N. Central America [in "State of the Climate in 2016"]. Bulletin of the American Meteorological Society, v. 98, n. 8, p. S180S183, 2017.

AMADOR, J.A.; RIVERA, E.R.; DURÁN-QUESADA, A.M.; MORA, G.; SÁENZ, F.; CALDERÓN, B.; MORA, N. The Easternmost Tropical Pacific. Part I: A Climate Review. Revista de Biología Tropical, v. 64, n. 1, p. 1-22, 2016 a.

AMADOR, J.A.; DURÁN-QUESADA, A.M.; RIVERA, E.R.; MORA, G.; SÁENZ, F.; CALDERÓN, B.; MORA, N. The Easternmost Tropical Pacific. Part II: Seasonal and Intraseasonal Modes of Atmospheric Variability. Revista de Biología Tropical, v. 64, n. 1, p. 23-57, 2016 b.

ANTHES, R.A. Development of asymmetries in a three-dimensional numerical model of the tropical cyclone. Monthly Weather Review, v. 100, n. 6, p. 461-476, 1972.

ARISCAIN, V. Nicaragua y Costa Rica: Huracán Otto: Movilización temprana fortaleció capacidad de respuesta de servicios de salud en Nicaragua y Costa Rica. Desastre, n. 193, 2017.

BENGTSSON, L.; HAGEMANN, S.; HODGES, K.I. Can climate trends be calculated from reanalysis data? Journal of Geophysical Research: Atmospheres, v. 109, n. D11, 2004.

BROWN, D. Hurricane Otto 20-26 November 2016. Tropical Cyclone Report. United States: National Hurricane Center, 2017.

BÜRGESSER, R.E. Assessment of the World Wide Lightning Location Network (WWLLN) detection efficiency by comparison to the Lightning Imaging Sensor (LIS). Quarterly Journal of the Royal Meteorological Society, v. 143, n. 708, p. 2809-2817, 2017.

BURPEE, R.W. The origin and structure of easterly waves in the lower troposphere of North Africa. Journal of the Atmospheric Sciences, v. 29, n. 1, p. 77-90, 1972.

CALVO SOLANO, O.D.; QUESADA HERNÁNDEZ, L.E.; HIDALGO LEÓN, H.G.; GOTLIEB, Y. Impactos de las sequías en el sector agropecuario del Corredor Seco Centroamericano. Agronomía Mesoamericana, v. 29, n. 3, p. 695-709, 2018.

CECIL, D.J.; ZIPSER, E.J.; NESBITT, S.W. Reflectivity, ice scattering, and lightning characteristics of hurricane eyewalls and rainbands. Part I: Quantitative description. Monthly Weather Review, v. 130, p. 16, 2002.
COLlIER, A.; HUGHES, A. lightning and the African ITCZ. Journal of Atmospheric and Solar-Terrestrial Physics, v. 73, p. 2392-2398, 2011.

COLLIER, A.; LICHTENBERGER, S.B.; DOWNS, J.; RODGER, C.; STEINBACH, P.G.M. Global lightning distribution and whistlers observed at Dunedin, New Zealand. Annales Geophysicae, v. 28, p. 499-513, 2010.

CCAD. Atlas Centroamericano para la Gestión Sostenible del Territorio. San Salvador, El Savador: Comisión Centroamericana de Ambiente y Desarrollo, Programa Regional para la Reducción de la Vulnerabilidad y Degradación Ambiental, Sistema de la Integración Centroamericana, 2011.

CORBOSIERO, K.L.; MOLINARI, J. The Relationship between storm motion, vertical wind shear, and convective asymmetries in tropical cyclones. Journal of the Atmospheric Sciences, v. 60, n. 2, p. 366-376, 2003.

DEMARIA, M.; DEMARIA, R.T.; KNAFF, J.A.; MOLENAR, D. Tropical cyclone lightning and rapid intensity change. Monthly Weather Review, v. 140, n. 6, p. 1828-1842, 2012.

ELSBERRY, R.L.; HARR, P. Tropical cyclone structure (TCS0field 8) experiment science basis, observational platforms, and strategy. Asia-Pacific Journal of Atmospheric Sciences, v. 44, p. 1-23, 2008.

HALPERIN, D.J.; FUELBERG, H.E.; HART, R.E.; COSSUTH, J.H.; SURA, P.; PASCH, R.J. An evaluation of tropical cyclone genesis forecasts from global numerical models. Weather and Forecasting, v. 28, n. 6, p. 1423-1445, 2013.

HIDALGO, H.G.; DURÁN-QUESADA, A.M.; AMADOR, J.A.; ALFARO, E.J. The Caribbean low-level jet, the intertropical convergence zone and precipitation patterns in the intra-Americas sea: A proposed dynamical mechanism. Geografiska Annaler: Series A, Physical Geography, v. 97, n. 1, p. 41-59, 2015.

HIDALGO, H.G.; ALFARO, E.J.; AMADOR, J.A.; BASTIDAS, A. Precursors of quasi-decadal dry-spells in the Central America dry corridor. Climate Dynamics, v. 53, n. 3, p. 1307-1322, 2019.

IMN. Huracán Otto. Informe Técnico. Costa Rica: Instituto Meteorológico Nacional, 2017.

IWASAKI, H. Climatology of global lightning classified by stroke energy using WWLLN Data. International Journal of Climatology, v. 35, n. 14, p. 4337-4347, 2015.

JENSENIUS JR, J.S. A detailed analysis of lightning deaths in the United States from 2006 through 2014. Executive Summary. United States: National Weather Service, 2015.

KOCHTUBAJDA, B.; STEWART, G.; FLANNIGAN, M. Summer convection and lightning over the Mackenzie River Basin and their impacts during 1994 and 1995. Atmosphere-Ocean, v. 40, p. 199-220, 2002.

KUO, H.L. On formation and intensification of tropical cyclones through latent heat release by cumulus convection. Journal of the Atmospheric Sciences, v. 22, n. 1, p. 40-63, 1965.

KURIHARA, Y.; TULEYA, R.E. Structure of a tropical cyclone developed in a three-dimensional numerical simulation model. Journal of the Atmospheric Sciences, v. 31, n. 4, p. 893-919, 1974. 
LANG, T.J.; PÉDEBOY, S.; RISON, W.; CERVENY, R.S.; MONTANYÀ, J.; CHAUZY, S.; MACGORMAN, D.R.; HOLLE, R.L.; ÁVILA, E.E.; ZHANG, Y.; CARBIN, G.; MANSELL, E.R.; KULESHOV, Y.; PETERSON, T.C.; BRUNET, M.; DRIOUECH, F.; KRAHENBUHL, D.S. WMO world record lightning extremes: Longest reported flash distance and longest reported flash duration. Bulletin of the American Meteorological Society, v. 98, n. 6, p. 1153-1168, 2016.

LATHAM, D.; WILLIAMS, E. Lightning and forest fires. In: Forest Fires: Behavior and Ecological Effects. San Francisco: Elsevier, 2001. p. 376-414.

LAWRENCE, M.B.; GROSS, J.M. Atlantic hurricane season of 1988. Monthly Weather Review, v. 117, n. 10, p. 22482259, 1989.

LIZANO, O.; SOTO, R. Caracterización de manglares de Centroamérica con sensores remotos. Revista de Biologia Tropical, v. 49, p. 331-340, 2001.

LYONS, W.A.; KEEN, C.S. Observations of lightning in convective supercells within tropical storms and hurricanes. Monthly Weather Review, v. 122, n. 8, p. 1897-1916, 1994.

MAGAÑA, V.; AMADOR, J.A.; MEDINA, S. The midsummer drought over Mexico and Central America. Journal of Climate, v. 12, n. 6, p. 1577-1588, 1999.

MALDONADO, T.; RUTGERSSON, A.; AMADOR, J.; ALFARO, E.; CLAREMAR, B. Variability of the Caribbean low-level jet during boreal winter: Large-scale forcings. International Journal of Climatology, v. 36, n. 4, p. 1954-1969, 2016.

MOLINARI, J.; MOORE, P.K.; IDONE, V.P.; HENDERSON, R.W.; SALJOUGHY, A.B. Cloud-to-ground lightning in hurricane Andrew. Journal of Geophysical Research: Atmospheres, v. 99, n. D8, p. 16665-16676, 1994.

MOLINARI, J.; MOORE, P.; IDONE, V. Convective structure of hurricanes as revealed by lightning locations. Monthly Weather Review, v. 127, n. 4, p. 520-534, 1999.

MUELLER, K.J.; DEMARIA, M.; KNAFF, J.; KOSSIN, J.P.; VONDER HAAR, T.H. Objective estimation of tropical cyclone wind structure from infrared satellite data. Weather and Forecasting, v. 21, n. 6, p. 990-1005, 2006.

MURAKAMI, H. Tropical cyclones in reanalysis data sets. Geophysical Research Letters, v. 41, n. 6, p. 2133-2141, 2014.

NICORA, M.; BÜRGESSER, R.; ROSALES, A.; QUEL, E.; ÁVILA, E. Actividad eléctrica asociada a la erupción del complejo volcánico Cordón Caulle durante 2011. Meteorologica, v. 38, n. 2, p. 121-131, 2013.

OLANDER, T.L.; VELDEN, C.S. The Advanced Dvorak Technique: Continued development of an objective scheme to estimate tropical cyclone intensity using geostationary infrared satellite imagery. Weather and Forecasting, v. 22, n. 2, p. 287-298, 2007.

PAN, L.; QIE, X.; LIU, D.; WANG, D.; YANG, J. The lightning activities in super typhoons over the Northwest Pacific. Science China Earth Science, v. 53, p. 1241-1248, 2010.

PASCH, R.J.; AVILA, L.A. Atlantic Hurricane Season of 1996. Monthly Weather Review, v. 127, n. 5, p. 581-610, 1999.
PEÑA, M. Characteristics of wet and dry spells over the Pacific side of Central America during the rainy season. Monthly Weather Review, v. 130, n. 12, p. 3054-3073.2002.

PERALTA RODRÍGUEZ, O.; CARRAZÓN ALOCÉN, J.; ZELAYA ELVIR, C.A. Buenas prácticas para la seguridad alimentaria y la gestión de riesgo. Organización de las Naciones Unidas para la Alimentación y la Agricultura (FAO), v. 53, n. 1, 2012.

RAGA, G.B.; DE LA PARRA, M.G.; KUCIENSKA, B. Deaths by lightning in Mexico (1979-2011): Threat or vulnerability? Weather, Climate, and Society, v. 6, n. 4, p. 434444, 2014.

RIBEIRO, W.; DE SOUZA, J.R.; LOPES, M.; CAMARA, R.; ROCHA, E.; ALMEIDA, A. Lightning and precipitation produced by severe weather systems over Belém, Brazil. Revista Brasileira de Meteorologia, v. 29, n. esp., p. 4159, 2014.

RODGER, C.; BRUNDELL, J.; DOWDEN, R. Location accuracy of VLF World-Wide Lightning Location (WWLL) network: Post-algorithm upgrade. Annales Geophysicae, v. 23, p. 277-290, 2005.

RODGER, C.J.; BRUNDELL, J.B.; HOLZWORTH, R.H.; LAY, E.H. Growing Detection efficiency of the world wide lightning location network. AIP Conference Proceedings, v. 1118, n. 1, p. 15-20, 2009.

RODGER, C.; WERNER, S.; BRUNDELL, J.; LAY, E.; THOMSON, N.; HOLZWORTH, R.; DOWDEN, R. Detection efficiency of the VLF World-Wide Lightning Location Network (WWLLN): Initial case study. Annales Geophysicae, v. 24, p. 3197-3214, 2006.

RUDLOSKY, S.D.; SHEA, D.T. Evaluating WWLLN Performance Relative to TRMM/LIS. Geophysical Research Letters, v. 40, n. 10, p. 2344-2348, 2013.

SADIGHI, S. Initiation of Streamers from Thundercloud Hydrometeors and Implications to Lightning Initiation. Melbourne, Florida: College of Science at Florida Institute of Technology, 2015.

SÁENZ SOTO, F.; AMADOR ASTÚA, J.A. Características del ciclo diurno de precipitación en el Caribe de Costa Rica. Revista de climatología, v. 16, p. 21-34, 2016.

SERRA, Y.; JIANG, X.; TIAN, B.; AMADOR, J.; MALONEY, E.; KILADIS, G. Tropical intraseasonal modes of the atmosphere. Annual Review of Environment and Resources, v. 39, n. 1, p. 189-215, 2014.

STEVENSON, S.N.; CORBOSIERO, K.L.; DEMARIA, M.; VIGH, J.L. A 10-Year survey of tropical cyclone inner-core lightning bursts and their relationship to intensity change. Weather and Forecasting, v. 33, n. 1, p. 23-36, 2017.

TAKAHASHI, T. Riming electrification as a charge generation mechanism in thunderstorms. Journal of the Atmospheric Sciences, v. 35, n. 8, p. 1536-1548, 1978.

THOMPSON, K.B.; BATEMAN, M.G.; CAREY, L.D. A Comparison of Two Ground-Based Lightning Detection Networks against the Satellite-Based Lightning Imaging Sensor (LIS). Journal of Atmospheric and Oceanic Technology, v. 31, n. 10, p. 2191-2205, 2014.

VINCENT, E.M.; LENGAIGNE, M.; MENKES, C.E.; JOURDAIN, N.C.; MARCHESIELLO, P.; MADEC, G. Interannual variability of the South Pacific convergence zone 
and implications for tropical cyclone genesis. Climate Dynamics, v. 36, n. 9, p. 1881-1896, 2011.

\section{Sitios web}

CIMSS, http://cimss.ssec.wisc.edu/goes/blog/archives/22683

NESDIS, https://www.nesdis.noaa.gov/sites/default/files/assets/ images/hurricane_ss_scale.jpg.

NHC, https://www.nhc.noaa.gov/aboutsshws.php
RAMMB， http://rammb.cira.colostate.edu/research/tropical_cy clones/tc_extended_best_track_dataset/data/ebtr $\mathrm{k}$ atlc 1988 2016.txt

WWLLN, http://wwlln.net/new/map/

License information: This is an open-access article distributed under the terms of the Creative Commons Attribution License (type CC-BY), which permits unrestricted use, distribution and reproduction in any medium, provided the original article is properly cited. 